\title{
Influence on Deep Brain Stimulation from Lead Design, Operating Mode and Tissue Impedance Changes - A Simulation Study
}

Fabiola Alonso', Simone Hemm-Ode ${ }^{1,2}$ and Karin Wårdell ${ }^{*}$

${ }^{1}$ Department of Biomedical Engineering, Linköping University, Sweden

${ }^{2}$ University of Applied Sciences and Arts Northwestern Switzerland, School of Life Sciences, Institute for Medical and Analytical Technologies, Switzerland

\begin{abstract}
Background: Deep brain stimulation (DBS) systems in current mode and new lead designs are recently available. To switch between DBS-systems remains complicated as clinicians may lose their reference for programming. Simulations can help increase the understanding.

Objective: To quantitatively investigate the electric field (EF) around two lead designs simulated to operate in voltage and current mode under two time points following implantation.

Methods: The finite element method was used to model Lead 3389 (Medtronic) and 6148 (St Jude) with homogenous surrounding grey matter and a peri-electrode space (PES) of $250 \mu \mathrm{m}$. The PES-impedance mimicked the acute (extracellular fluid) and chronic (fibrous tissue) time-point. Simulations at different amplitudes of voltage and current $(n=236)$ were performed using two different contacts. Equivalent current amplitudes were extracted by matching the shape and maximum EF of the $0.2 \mathrm{~V} / \mathrm{mm}$ isolevel.

Results: The maximum EF extension at $0.2 \mathrm{~V} / \mathrm{mm}$ varied between 2-5 $\mathrm{mm}$ with a small difference between the leads. In voltage mode EF increased about $1 \mathrm{~mm}$ at acute compared to the chronic PES. Current mode presented the opposite relationship. Equivalent EFs for lead 3389 at $3 \mathrm{~V}$ were found for $7 \mathrm{~mA}$ (acute) and $2.2 \mathrm{~mA}$ (chronic).

Conclusions: Simulations showed a major impact on the electric field extension between postoperative time points. This may explain the clinical decisions to reprogram the amplitude weeks after implantation. Neither the EF extension nor intensity is considerably influenced by the lead design. However, the EF distribution is affected by the larger contact of Lead 6148 generating an electric field below the tip.
\end{abstract}

Keywords: Deep brain stimulation; Voltage and current stimulation; Finite element method; Lead design; Peri-electrode space

\section{Introduction}

Deep brain stimulation (DBS) is an established therapy for movement disorders such as Parkinson's disease (PD), essential tremor and dystonia [1]. To date more than 120,000 patients have received DBS implants. During the first two decades of the modern DBS era all implantations were performed using the same type of stimulation control and lead configuration; a four contact lead connected to a voltage controlled neurostimulator. In 2009 the first current controlled DBS device was introduced in Europe on PD patients. Today several DBS lead designs and stimulation modes are available but still not fully established as clinical alternatives. Furthermore intensive research is being performed to develop new DBS systems, e.g. for steering of the simulation field [2-5].

Present literature seems to show that current controlled stimulation induces at least as good clinical effects as voltage controlled stimulation $[6,7]$, and that it should be preferred towards voltage controlled devices due to the automatic voltage adjustment as impedance changes $[8,9]$. Nevertheless, to switch from one mode to the other remains complicated as clinicians risk to lose their reference for programming. The same is valid for switching from one commercially available lead design to another as the differences or the influence of the design on voltage, current or electric field distribution has not been discussed so far to our knowledge. In addition to questions concerning the design, neurologists often have to deal with changes in impedance during postoperative patient management, not only due to hardware complications [10], but due to tissue changes around the electrode [11-13].
A way to try to provide answers to the above formulated questions concerning different DBS lead designs, stimulation modes and influence of impedance changes is to use computer models to simulate the electrical behaviour around active DBS-contacts. Current controlled stimulation systems have not been studied as much as voltage-controlled systems. The few studies published rely on in vivo experimental recordings performed in animals [9] or experiments where the brain tissue is not included in the study [14]. Computational model studies have been designed to analyse the influence of different pulse waveforms or electrode-tissue interface considering both voltage and current controlled stimulation [15-17].

Our group has previously used the finite element method (FEM) to investigate the influence on the DBS fields from tissue types such as cystic cavities [18] and white matter heterogeneity and anisotropy [19]. Also, patient-specific models and simulations of DBS have been used to increase the understanding of the response to stimulation for a number of targets including the subthalamic nucleus [20-23] the globus

*Corresponding author: Karin Wårdell, Department of Biomedical Engineering Linköping University, 58185 Linköping, Sweden, Tel: +46 10 1030000; E-mail: karin.wardell@liu.se

Received April 02, 2015; Accepted June 03, 2015; Published June 08, 2015

Citation: Alonso F, Hemm-Ode S, Wårdell K (2015) Influence on Deep Brain Stimulation from Lead Design, Operating Mode and Tissue Impedance Changes A Simulation Study. Brain Disord Ther 4:169. doi:10.4172/2168-975X.1000169

Copyright: @ 2015 Alonso F, et al. This is an open-access article distributed under the terms of the Creative Commons Attribution License, which permits unrestricted use, distribution, and reproduction in any medium, provided the original author and source are credited.t 
pallidus internus [24-27], and the ventral intermedius nucleus of the thalamus for essential tremor [28]. Such patient-specific simulations should preferable take into account both, the tissue's heterogeneity (e.g. differences between grey and white matter) and the anisotropy of the white matter [19]. When studying the differences between lead designs and stimulation modes however, it is an advantage to reduce the number of parameters that can influence the outcome. Especially when comparing different stimulation modes and lead designs, the relative difference is most important and not the actual extent of the stimulation. Therefore a homogenous tissue model with impedance often mimicking grey tissue, is preferred [18] and also used for the present study.

Stimulation results may be represented and visualized by different electrical quantities such as the electric field (EF) [21,24,25], the second difference of the electric potential $[3,4]$, and the volume of tissue activated derived from neuron models coupled to finite element simulations $[17,20,29]$. By using a fixed isolevel the EF can be used for relative comparisons between simulations, and also allow for visualization directly in the $\mathrm{mm}$-scale. The electric field is also the entity which shows the least influence on the activation threshold caused by change in axon diameter, pulse width and amplitude [29]. Therefore EF is a suitable entity for the present study, where the aim is to investigate the influence on the electric field from two DBS-lead designs, two operating modes i.e. current or voltage stimulation, and the impedance in the peri-electrode space (PES). The PES was set to mimic two post-operative time points: the acute stage corresponding to leakage of extracellular fluid and a chronic condition related to fibrous tissue [30].

\section{Materials and Methods}

\section{DBS leads and brain models}

The investigation was based on two different DBS lead designs: Lead 3389 (Medtronic Inc., USA) (Figure 1a) and Lead 6148 (St Jude Medical Inc., USA) (Figure 1b). 2D axisymmetric FEM models were developed using COMSOL Multiphysics Version 4.3a (Comsol Multiphysics AB, Sweden). The geometry of the leads is based on the manufacturers' respective technical specifications and consists in four platinum iridium alloy (Pt/Ir) contacts separated by $0.5 \mathrm{~mm}$ of insulation. The contacts are $1.5 \mathrm{~mm}$ long except for Lead 6148's distal contact which is $3 \mathrm{~mm}$ long and covers the tip of the lead. The diameter of Lead 3389 is $1.27 \mathrm{~mm}$ while it is $1.4 \mathrm{~mm}$ for Lead 6148 (Figure 1). The brain tissue surrounding the lead was modelled as a homogeneous and isotropic medium in a rectangular shape $(60 \mathrm{~mm} \times 56 \mathrm{~mm})$, with electrical conductivity and relative permittivity corresponding to grey matter $(\sigma=0.09 \mathrm{~S} / \mathrm{m}$ and $\in=3.9 \times 106$ [31] $)$. A PES of $250 \mu \mathrm{m}$ was added to the models in order to mimic the interface between the electrode and the brain tissue at two different time points post DBS-implantation. For the acute stage, which simulates the leakage of extracellular fluid provoked immediately after leads insertion, the electrical conductivity and relative permittivity were set to values corresponding to cerebral spinal fluid (CSF) $\sigma=2 \mathrm{~S} / \mathrm{m}$ and $\in=109$ respectively [31]. The chronic stage, where fibrous tissue covers the electrode surface a few weeks after surgery, was mimicked by using $\sigma=0.06 \mathrm{~S} / \mathrm{m}$ and $\epsilon=1.7 \times 106$ instead for the PES [32].

\section{Governing equation and boundary conditions}

The boundary conditions for the leads were set in accordance with a monopolar configuration which considers the active contact as the cathode, and the outer boundaries of the tissue as the anode representing the ground from the impulse generator. The boundary for the active contact was set as either a voltage or current ideal source. The non-active contacts were assigned to floating potential $[26,33]$ and the spaces between the contacts were set as electric insulation. The electric field was measured at different points in front of the lead using different mesh densities and stimulated region sizes in order to assure that neither the chosen boundaries of the stimulation region nor the mesh density affected the electric field value. The mesh applied was set to the finest resolution available with a denser distribution of elements around the leads. This corresponded to 28297 elements with a minimum element size of $0.0012 \mathrm{~mm}$ and an average element quality of 0.9824 . The simulations were performed using electric currents physics interface which is able to model electric currents in conductive media; the electric field distribution around the DBS electrode was obtained

\section{Lead 3389}

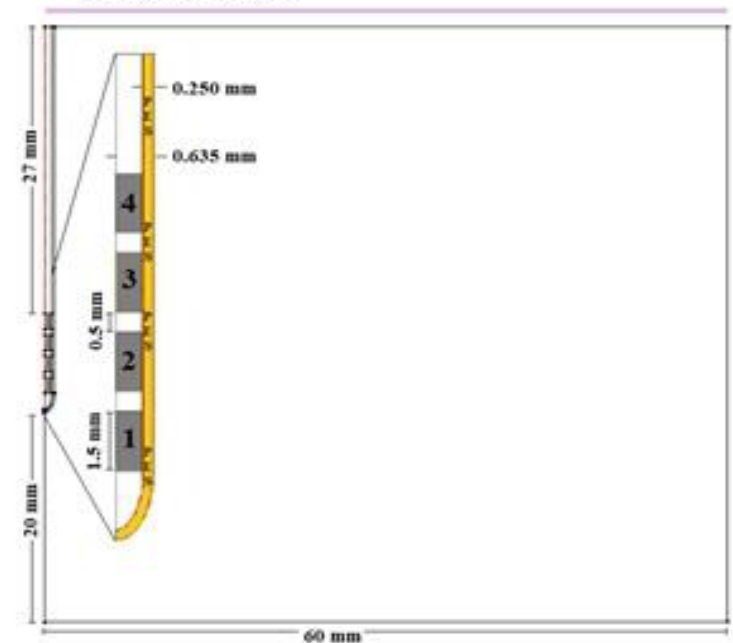

Lead 6148

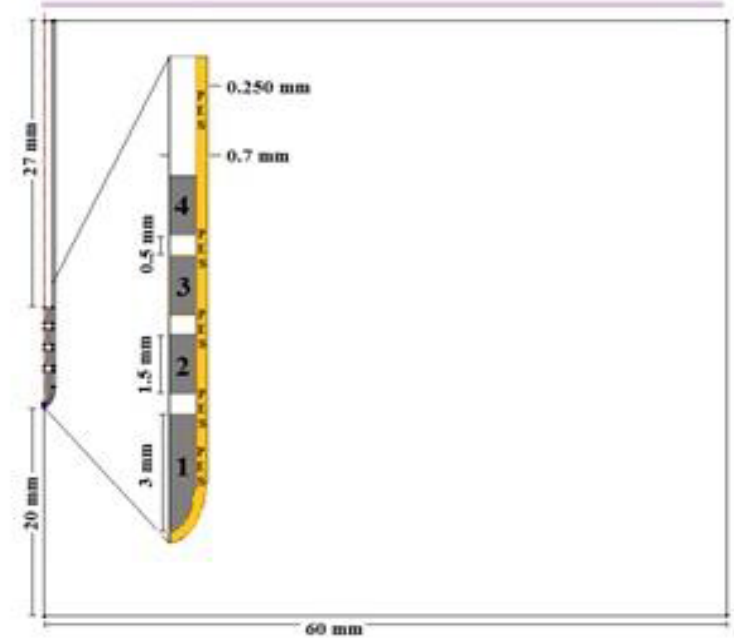

Figure 1: 2D axisymmetric models of Lead 3389 (a) and Lead 6148 (b) surrounded by homogeneous and isotropic medium representing grey matter and a $250 \mu$ m thick peri-electrode space (PES). 
using the COMSOL MUltifrontal Massively Parallel sparse direct Solver (MUMPS). The electric field generated is calculated using the equation of continuity for steady currents:

$$
\nabla \cdot J=-\nabla \cdot(\sigma \nabla \mathrm{V})=0\left[\mathrm{~A} / \mathrm{m}^{3}\right](1)
$$

Where $\nabla \bullet$ is the divergence, J the current density, $\nabla$ the gradient, $\mathrm{V}$ the electric potential and $\sigma$ the electrical conductivity. More details regarding the modelling and simulation principle can be found in $[18,21,34]$.

\section{Simulations}

Two investigations were performed. The first included both leads under voltage controlled stimulation (leads comparison). In this setting simulations $(\mathrm{n}=32)$ were performed for acute and chronic scenarios for both leads using the first (lower) and the third (second upper) contacts. The stimulation amplitudes applied were $1-4 \mathrm{~V}$ in steps of $1 \mathrm{~V}$. For the second examination only one of the leads (3389) was considered using both voltage and current controlled stimulation (operating modes comparison). Equivalent current values capable to generate exactly the same $\mathrm{EF}$ distribution as the voltage stimulation i.e. 1, 2, 3, $4 \mathrm{~V}$ were found by performing a parametric sweep from 0.8 to $10 \mathrm{~mA}$ in steps of $0.1 \mathrm{~mA}(\mathrm{n}=204)$.

\section{Data analysis}

The EF was visualized and analysed using an isolevel of $0.2 \mathrm{~V} / \mathrm{mm}$ $[22,24,27]$. The maximum EF extension of this isolevel was measured for all voltage simulations and plotted against the respective lead and contact. A fixed amplitude of $3 \mathrm{~V}$ was used to present the results and for further investigation of the electric field's shape, extension and intensity between leads. The EF intensity was measured by placing 16 evaluation points parallel to the leads with a separation of $0.5 \mathrm{~mm}$ between each other. This measurement was performed at 1,3 and $5 \mathrm{~mm}$ from the leads' surface. Equivalent current amplitudes for Lead 3389, resulting in the same EF distribution and shape as obtained with 1, 2, 3 and $4 \mathrm{~V}$ under chronic conditions, were extracted. This was achieved displaying both types of stimulation isolevels simultaneously, and comparing them visually at each stimulation amplitude. The current value which isolevel overlapped the isolevel for voltage stimulus was considered the equivalent current amplitude.

\section{Results}

\section{Leads comparison}

In Figure 2 the distribution of the EF around the active contacts is presented for Lead 3389 (Figure 2a and 2b) and Lead 6148 (Figure 2c and $2 \mathrm{~d}$ ) as simulated for $3 \mathrm{~V}$, a PES of $250 \mu \mathrm{m}$ under acute and chronic conditions. The shape and maximal extension of the EF considerably differed between the two simulated time points for all contacts. At the acute stage, the EF was broadened below the tip in both leads regardless of the active contact. At the chronic stage, the EF was also distributed below the tip for Lead 6148 (Figure $2 \mathrm{c}$ and $2 \mathrm{~d}$ ). The maximum EF extension was about $1 \mathrm{~mm}$ greater at the acute time point compared to chronic conditions. Plots of the maximum EF extension as related to the four voltage stimulation amplitudes are presented in Figure 3 for each lead and contact.

Figure 4 presents the electric field intensity measured for $3 \mathrm{~V}$ at 1 $\mathrm{mm}$ from the contact surface. The evaluation was performed for both leads under the two tissue scenarios. The intensity at the acute stage was approximately $0.1 \mathrm{~V} / \mathrm{mm}$ larger. Both leads presented the highest EF
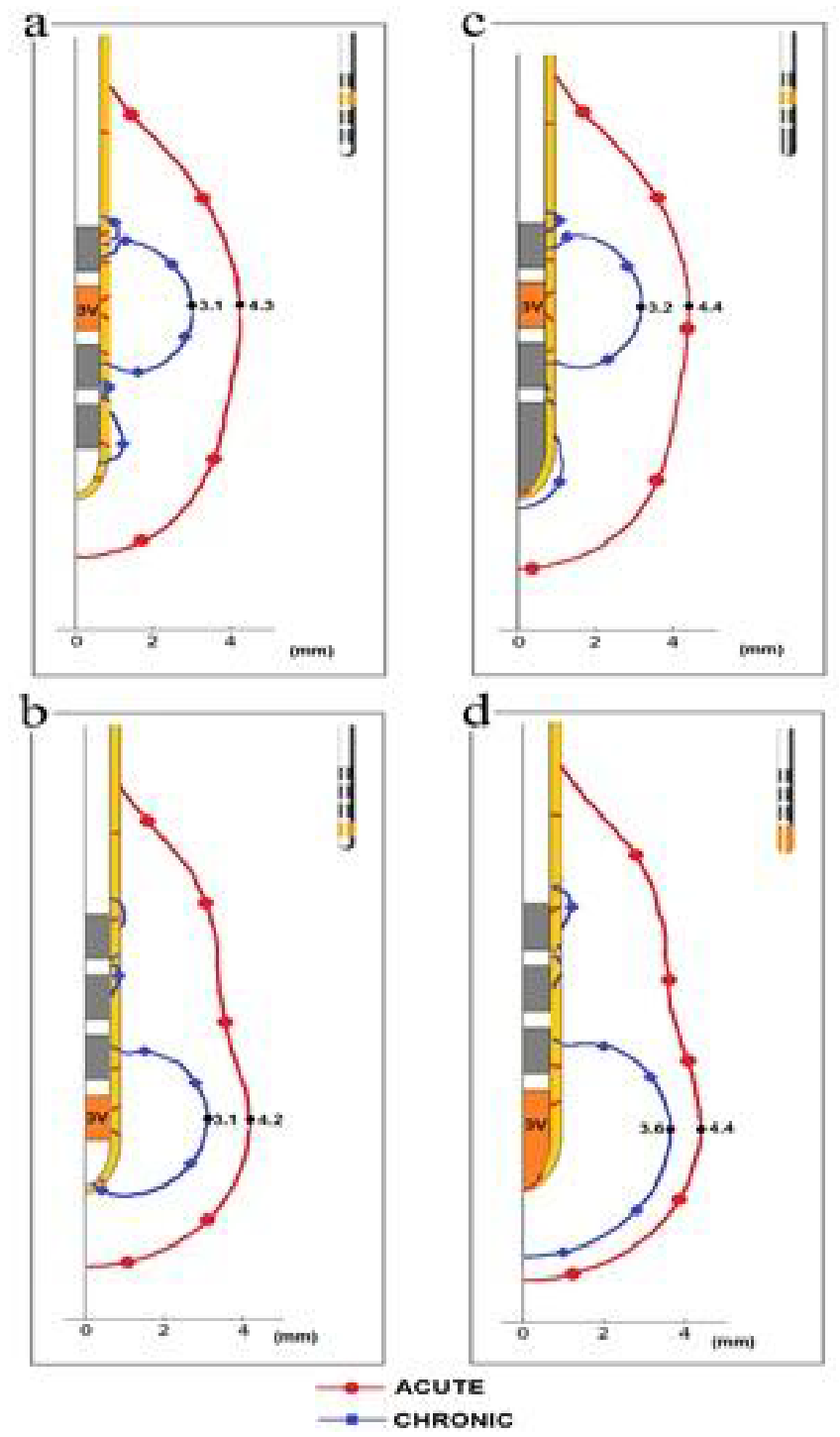

Figure 2: Electric field isolevels $(0.2 \mathrm{~V} / \mathrm{mm})$ obtained for voltage controlled stimulation $(3 \mathrm{~V})$ under acute and chronic conditions (red and blue lines respectively). Maximum EF extension shown in $\mathrm{mm}$ for each isolevel. Simulations performed with Lead 3389 ( $a$ and b) and Lead 6148 (c and d) models, using the third ( $a$ and $c$ ) and the first ( $b$ and $d$ ) contact as the active electrode.

intensity in front of the middle of the active contact. Similar behavior was found at the 3 and $5 \mathrm{~mm}$ distant evaluation points.

\section{Operating modes comparison}

Table 1 presents the equivalent current amplitude values to the commonly clinically used voltage values for both contacts, leads and the two different PES. The PES corresponding to the acute stage, required higher current stimulation settings in order to obtain the same electric field distribution as when using voltage controlled stimulation. As an example, equivalent $\mathrm{EFs}$ for $3 \mathrm{~V}$ were found with $7 \mathrm{~mA}$ (acute) and 2.2 $\mathrm{mA}$ (chronic) stimulation. Figure 5 presents a comparison of the EF isolevels using $3 \mathrm{~V}$ and $2.2 \mathrm{~mA}$ for both postoperative time points for Lead 3389. The electric field isolevels perfectly overlap in the chronic scenario (Figure $5 \mathrm{c}$ and $5 \mathrm{~d}$ ). In the acute stage, however, which has an impedance in the PES radically different from the surrounding grey 
Citation: Alonso F, Hemm-Ode S, Wårdell K (2015) Influence on Deep Brain Stimulation from Lead Design, Operating Mode and Tissue Impedance Changes - A Simulation Study. Brain Disord Ther 4:169. doi:10.4172/2168-975X.1000169

Page 4 of 8
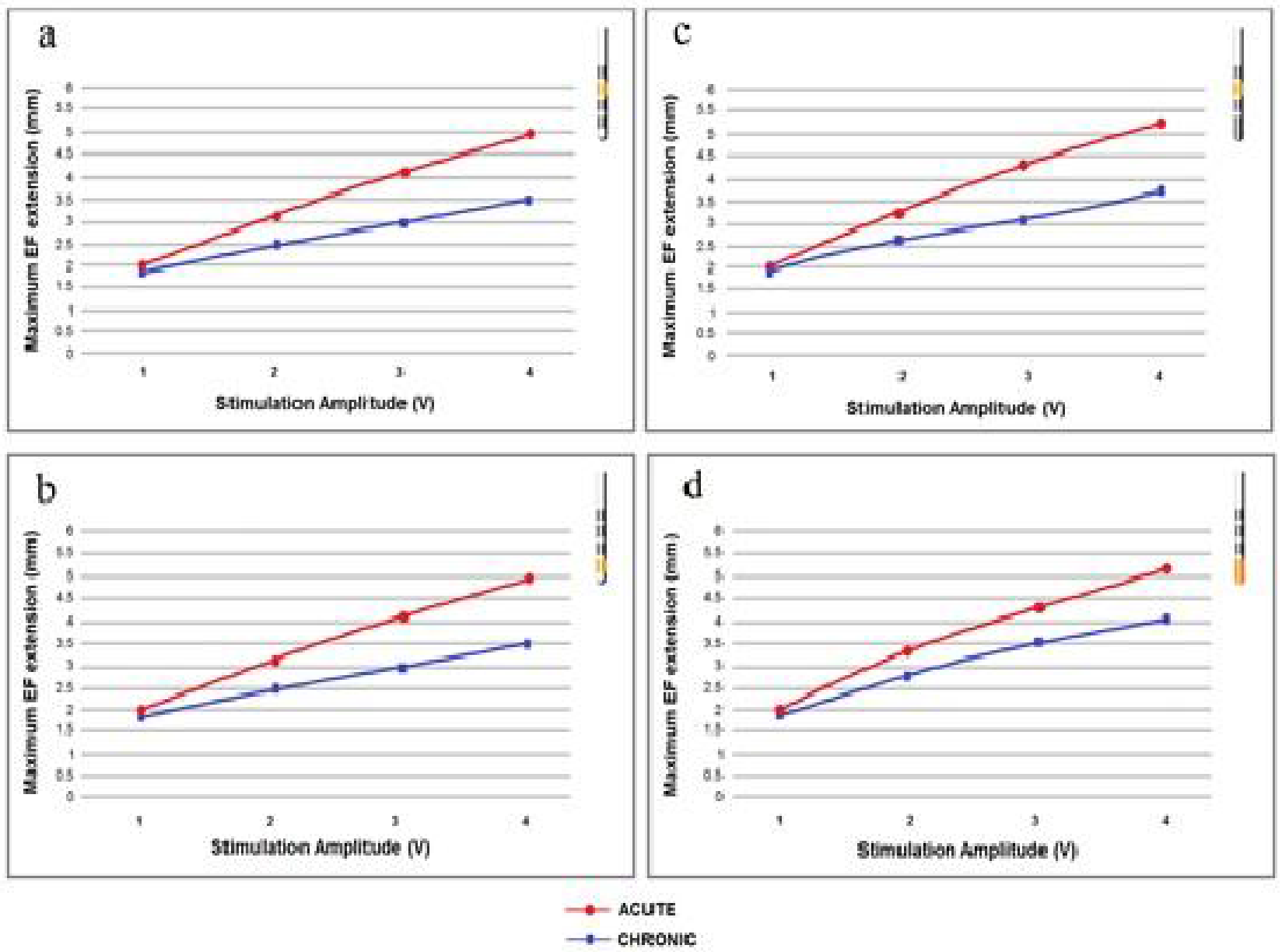

Figure 3: Relation between the maximum electric field extension and the voltage amplitudes for the third and first contacts of Lead 3389 (a and b) and Lead 6148 (c and d). Measurements obtained from simulations under acute and chronic conditions (red and blue lines respectively).

\begin{tabular}{|c|c|c|c|c|}
\hline \multirow{2}{*}{$\begin{array}{c}\text { Voltage } \\
\text { Stimulation } \\
\text { Amplitude (V) }\end{array}$} & \multicolumn{3}{|c|}{ Equivalent Current Stimulation Amplitude (mA) } \\
\cline { 2 - 5 } & \multicolumn{2}{|c|}{ Acute } & \multicolumn{2}{c|}{ Chronic } \\
\cline { 2 - 5 } & $\begin{array}{c}\text { LEAD 3389 } \\
\text { Third/First } \\
\text { Contact }\end{array}$ & $\begin{array}{c}\text { Third/First } \\
\text { Contact }\end{array}$ & $\begin{array}{c}\text { Third/First } \\
\text { Contact }\end{array}$ & $\begin{array}{c}\text { Third/First } \\
\text { Contact }\end{array}$ \\
\hline 1 & $2.3 / 2.3$ & $2.3 / 2.4$ & $0.7 / 0.7$ & $0.7 / 1.1$ \\
\hline 2 & $4.5 / 4.5$ & $4.5 / 4.7$ & $1.5 / 1.5$ & $1.5 / 2.2$ \\
\hline 3 & $7.0 / 7.0$ & $7.0 / 7.1$ & $2.2 / 2.2$ & $2.3 / 3.2$ \\
\hline 4 & $9.0 / 9.0$ & $9.0 / 9.5$ & $2.9 / 2.9$ & $3.0 / 4.3$ \\
\hline
\end{tabular}

Table 1: Current amplitude values for the third and first contacts with the best match to the commonly clinically used voltage amplitudes for Lead 3389 and Lead 6148 at two post-implantation stages.

matter a significant change in $\mathrm{EF}$ in both voltage and current modes is seen for both active contacts. The electric field is spread along the lead (Figure $5 \mathrm{a}$ and $5 \mathrm{~b}$ ). Compared to the chronic stimulation the electric field is also extending about $1 \mathrm{~mm}$ further away in voltage mode and about $1 \mathrm{~mm}$ shorter in current mode (Figure $5 \mathrm{a}$ and $5 \mathrm{~b}$ ). Figure 6 shows the relation between the maximum EF extension and the stimulation amplitudes for both current and voltage stimulations. For current controlled stimulation the EF extension increases in steps of about 0.5 $\mathrm{mm}$ for both time points. In voltage mode the EF extension had a higher increase with amplitude at the acute stage (Figure $6 \mathrm{a}$ and $6 \mathrm{~b}$ ). The EF intensities measured for voltage and current controlled stimulation are presented in Figure 7. The intensity obtained at the acute stage for current control (Figure $7 \mathrm{c}$ and $7 \mathrm{~d}$ ) is lower $(0.6 \mathrm{~V} / \mathrm{mm})$ than for voltage stimulation regardless of the contact used.

\section{Discussion}

In this computer simulations study the influence on the electric field from two different DBS-lead designs both operating in voltage and current modes have been compared. In order to mimic realistic time points, crucial in the postoperative DBS management, the impedance in the space around the electrodes was varied at the same time as grey matter was assumed as target tissue. For typical amplitudes used in the clinic, the simulations showed electric field extensions ranging from 2 to $5 \mathrm{~mm}$ which is in agreement with other studies [35].

\section{Leads comparison}

New DBS-lead designs are presently introduced in health care, and it is therefore imperative to increase the clinical knowledge about similarities and differences between different lead configurations. In general, the shape and maximum extension of the electric field were very similar for both leads in voltage mode, especially with a PES mimicking the leakage of extracellular fluid, i.e. the acute stage post implantation. When simulating the chronic time point, however, Lead 

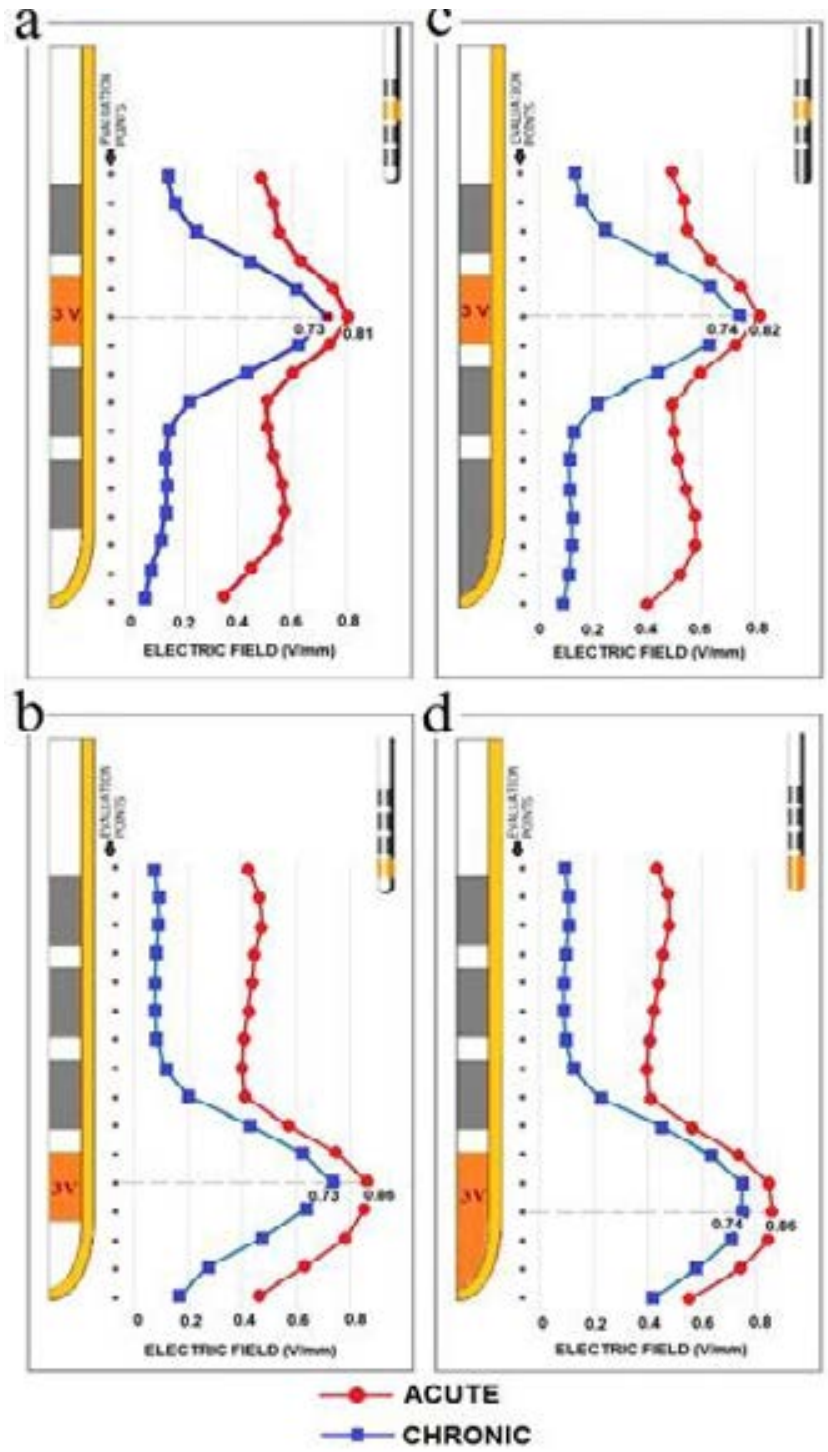

Figure 4: Electric field intensity evaluated at $1 \mathrm{~mm}$ from the electrode surface under voltage controlled stimulation ( $3 \mathrm{~V})$. a) Third and b) first contact of Lead 3389 and third and first of Lead 6148 (c and d). Measurements performed for acute and chronic conditions.

6148 exhibited an EF below the tip regardless of the active electrode. This behaviour indicates that additional tissue regions millimetres away from an active contact may be stimulated (Figure $2 \mathrm{c}$ and $2 \mathrm{~d}$ ). Lead 6148 is $0.13 \mathrm{~mm}$ thicker and its contact at the tip is twice the length hence it has a considerable larger area. While Lead 3389 required the same current amplitude regardless of the active contact, Lead 6148 needed a higher current to achieve the same EF when using the tip contact. For instance, the equivalent current amplitude for $3 \mathrm{~V}$ was $2.3 \mathrm{~mA}$ using the third contact but $3.2 \mathrm{~mA}$ for the first (Table 1). A larger contact reduces the current density and consequently the current applied has to be increased to achieve the same electric field.

\section{Operating mode}

Simulations showed that in voltage mode the EF extension is significantly enlarged when the PES has a lower impedance (higher conductivity) that the surrounding tissue as in the acute time point (Figure $5 \mathrm{a}$ and $5 \mathrm{~b}$ ). An explanation could be that due to the lower impedance the energy is not maintained in the PES but delivered further away i.e. similarly to a voltage divider between the PES and surrounding tissue compartments. A similar behaviour of the electric field was shown for FEM-simulated CSF-filled cystic cavities i.e. perivascular Virchow-Robin spaces, sometimes appearing in the pallidum [18]. On the contrary, in current mode a lower impedance in the PES reduces the voltage in that region in order to maintain the same current therefore shortening the EF based on Ohm's law. This behaviour is consistent with the results of the EF intensity (Figure 7) and with the corresponding adjustment of the current amplitude values shown in Table 1 . These results can be compared with the postoperative adjustment of the stimulation amplitude commonly done within a
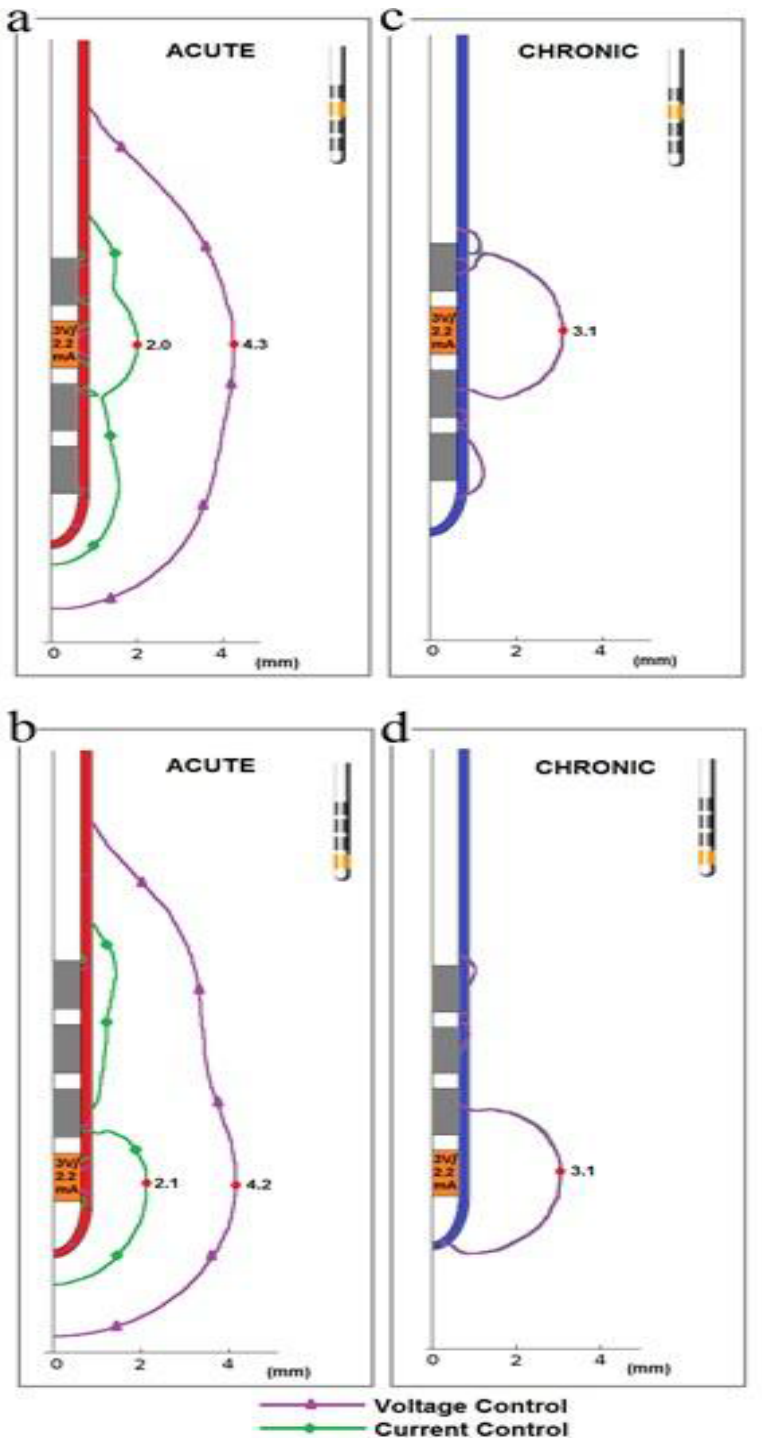

Figure 5: Electric field isolevels $(0.2 \mathrm{~V} / \mathrm{mm})$ for voltage and current controlled stimulation (purple and green lines respectively). Simulations performed with Lead 3389 model at acute ( $a$ and b) and chronic ( $c$ and d) stages. Maximum EF extension measured in $\mathrm{mm}$ is shown with red dots for (a and $\mathrm{c}$ ) the third and ( $b$ and $d$ ) first contact. 

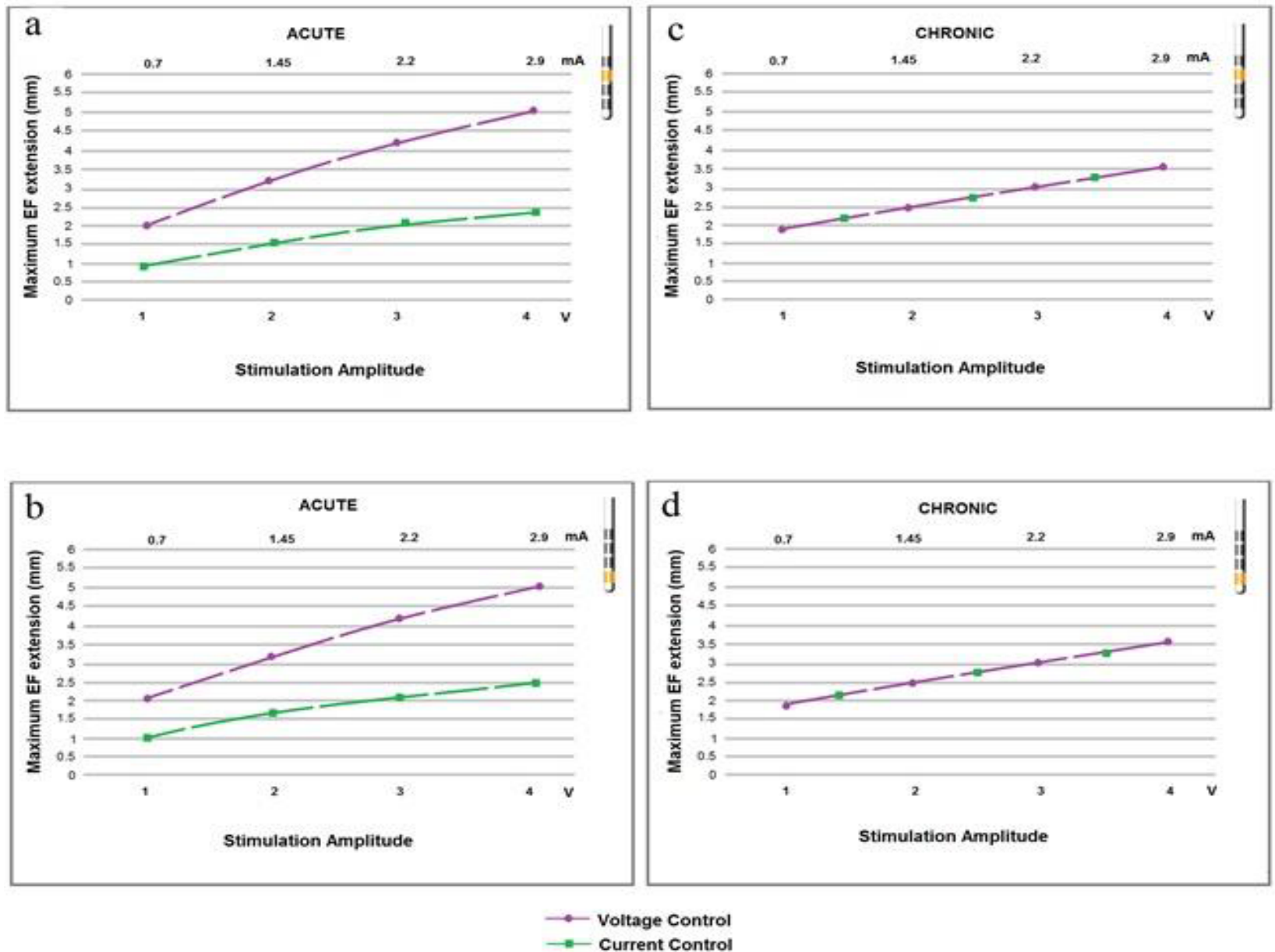

Figure 6: Relation between the maximum electric field extension and amplitude values of voltage and current controlled stimulation. Simulations performed using Lead 3389 under acute ( $a$ and $b$ ) and chronic ( $c$ and d) conditions for ( $a$ and $c$ ) third and (b and d) first contact.

few weeks following DBS implantation in Parkinson's disease [36]. Furthermore, the EF extension at $0.2 \mathrm{~V} / \mathrm{mm}$ obtained at different stimulation amplitudes (Figure 6) at chronic stage is comparable with the clinical neurophysiological stimuli-response studies which suggest a linear correlation between a stimulation threshold and distance, i.e. $3 \mathrm{~mA}$ corresponds to $3 \mathrm{~mm}[35,37,38]$. In vivo studies [9] and a recent review by Bronstein et al. [8] suggest the use of current controlled DBS systems based on their low susceptibility to the conductivity of the brain.

\section{Peri-electrode space thickness}

The thickness and change over time of the PES is debatable. Clinical [11] and animal [12] studies have reported, based on in-vivo measurements, that impedance increases following implantation, but that it rapidly decreases again to some extent during clinically relevant stimulation. Post-mortem studies $[39,40]$ have shown that there might be differences in the thickness of this region. Nielsen et al. found a 150 $\mu \mathrm{m}$ thick fibrillary gliosis layer around the electrode tract and Haberler and colleagues a layer less than $500 \mu \mathrm{m}$. In addition, these studies showed that the PES differed depending on the brain target investigated. In the present study a PES thickness between the values suggested by Nielsen and Haberler was selected and kept constant for comparative simulations. The selected PES thickness, $250 \mu \mathrm{m}$, has also been used in previous simulation studies by Yousif et al. [30]. In order to investigate the influence from the PES thickness additional simulations $(n=12)$ were performed for PES of $125 \mu \mathrm{m}, 250 \mu \mathrm{m}$ and $500 \mu \mathrm{m}$ at $3 \mathrm{~V}$ with the acute and chronic tissue settings using the third contact active for Lead 3389. By changing the PES thickness in the simulations it was found that there is a small $(\leq 0.3 \mathrm{~mm})$ influence on the electric field (Table 2$)$ when the thickness was doubled. The difference was more pronounced at the acute stage when the electric conductivity was set to CSF. By keeping as many parameters as possible constant, we could compare the leads and stimulation modes for a range of amplitudes and different post-operative mimicked tissue conditions $[17,41,42]$. Relative comparisons were possible by using a fixed EF isolevel, here set to $0.2 \mathrm{~V} / \mathrm{mm}$. This isolevel has been used in several previous investigations [22,24,27] and it has also been pointed out as a possible predictor of neural activation [29].

To gain more detailed knowledge about DBS and tissue interaction, future models and simulations could be made more complex, both regarding simulation of the $\mathrm{PES}$, and by using patient-specific information e.g. from MRI and diffusion tensor imaging as input $[21,22]$. Simulations can also be combined with axon cable models for increased knowledge of the influence from various axon diameter, as well as pulse width of the DBS-systems $[29,43]$. It should, however, be stressed that model based studies always contain simplifications of 
Citation: Alonso F, Hemm-Ode S, Wårdell K (2015) Influence on Deep Brain Stimulation from Lead Design, Operating Mode and Tissue Impedance Changes - A Simulation Study. Brain Disord Ther 4:169. doi:10.4172/2168-975X.1000169

Page 7 of 8

\begin{tabular}{|c|c|c|c|c|}
\hline \multirow{2}{*}{} & \multicolumn{4}{|c|}{ Distance from electrode surface (mm) } \\
\cline { 2 - 5 } & \multicolumn{2}{|c|}{ Voltage control } & \multicolumn{2}{c|}{ Current control } \\
\hline PES thickness $(\boldsymbol{\mu m})$ & Acute & Chronic & Acute & Chronic \\
\hline 125 & 4.0 & 3.1 & 2.4 & 3.3 \\
\hline 250 & 4.3 & 3.1 & 2.3 & 3.3 \\
\hline 500 & 4.6 & 3.0 & 2.0 & 3.3 \\
\hline
\end{tabular}

Table 2: Distance $(\mathrm{mm})$ reached from electrode surface by the electric field isoleve of $0.2 \mathrm{~V} / \mathrm{m}$ for different thicknesses peri-electrode space (PES) and Lead 3389 using the third contact. Simulations were done with $3 \mathrm{~V}$ and $2.5 \mathrm{~mA}$ for equivalent electric fields.
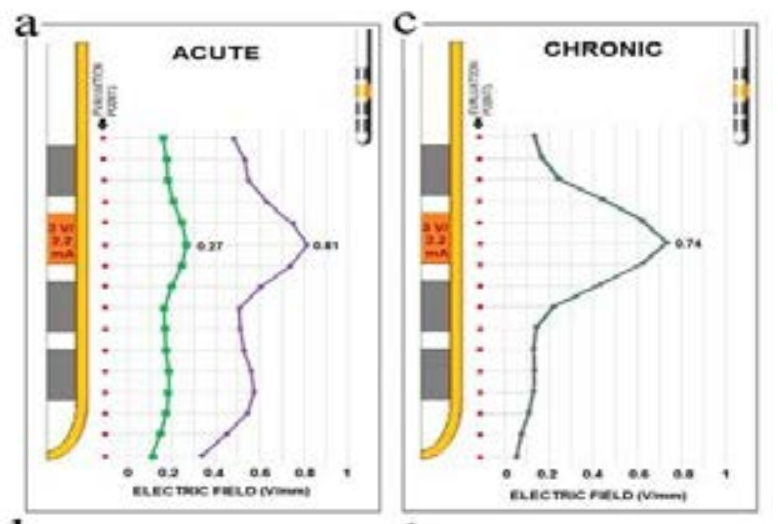

b
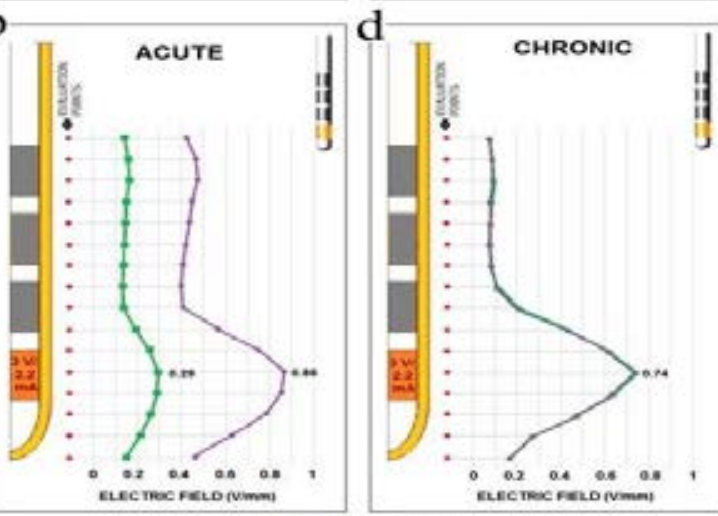

- Vousas Contrat

Figure 7: Electric field intensity evaluated at $1 \mathrm{~mm}$ from the electrode surface of Lead 3389. Simulations performed for voltage and current stimulation (purple and green lines respectively) under acute ( $a$ and b) and chronic (c and d) conditions.

real clinical situations [42], but nevertheless can serve as a guide for increased understanding of electric field spread in different clinical situations and comparisons between different leads, tissue conditions and stimulation modes.

\section{Conclusions}

FEM-simulations of the electric field in voltage and current modes for two DBS lead-designs have been performed at different time-points following implantation. The results show a significant influence from the peri-electrode space at the acute time point when impedance was lower (higher conductivity) than surrounding grey matter. In voltage mode, the electric field was broadened while in current mode shortened. These results can help to clarify the postoperative adjustments of the DBS-amplitudes often necessary a few weeks after implantation. The lead designs showed no relevant difference in the maximal electric field extension, nevertheless due to a larger contact at its tip, Lead 6148 spread the electric field below the lead at all stages independent of the active contact. This behaviour suggests a careful planning and parameter selection in order to achieve a predefined electric field magnitude and shape.

\section{Acknowledgements}

The study was financially supported by the Swedish Research Council (Grant No. 621-2013-6078) and The Parkinson foundation at Linköping University. The authors do not have financial interests or conflict of interest to the companies related to this study.

\section{References}

1. Hemm S, Wårdell K (2010) Stereotactic implantation of deep brain stimulation electrodes: a review of technical systems, methods and emerging tools. Med Biol Eng Comput 48: 611-624.

2. Åström M, Tripoliti E, Zrinzo L, Martinez-Torres I, Limousin P, et al. (2008) Voltage steering to control deep brain stimulation-induced speech deficits. Acta Neurochirurgica Springer 953-954.

3. Butson CR, Mclntyre CC (2008) Current steering to control the volume of tissue activated during deep brain stimulation. Brain Stimul 1: 7-15.

4. Martens HC, Toader E, Decré MM, Anderson DJ, Vetter R, et al. (2011) Spatia steering of deep brain stimulation volumes using a novel lead design. Clin Neurophysiol 122: 558-566.

5. Liu HG, Ma Y, Zhang K, Ge M, Meng FG, et al. (2013) Subthalamic deep brain stimulation with a new device in Parkinson's disease: an open-label trial Neuromodulation 16: 212-218.

6. Ramirez de Noriega F, Eitan R, Marmor O, Lavi A, Linetzky E, et al. (2015) Constant Current versus Constant Voltage Subthalamic Nucleus Deep Brain Stimulation in Parkinson's Disease. Stereotact Funct Neurosurg 93: 114-121.

7. Lettieri C, Rinaldo S, Devigili G, Pisa F, Mucchiut M, et al. (2015) Clinical outcome of deep brain stimulation for dystonia: constant-current or constantvoltage stimulation? A non-randomized study. Eur J Neurol 22: 919-926.

8. Bronstein JM, Tagliati M, McIntyre C, Chen R, Cheung T, et al. (2015) The rationale driving the evolution of deep brain stimulation to constant-current devices. Neuromodulation 18: 85-88.

9. Lempka SF, Johnson MD, Miocinovic S, Vitek JL, Mclntyre CC (2010) Currentcontrolled deep brain stimulation reduces in vivo voltage fluctuations observed during voltage-controlled stimulation. Clin Neurophysiol 121: 2128-2133.

10. Farris S, Vitek J, Giroux ML (2008) Deep brain stimulation hardware complications: the role of electrode impedance and current measurements. Mov Disord 23: 755-760.

11. Hemm S, Vayssiere N, Mennessier G, Cif L, Zanca M, et al. (2004) Evolution of Brain Impedance in Dystonic Patients Treated by GPi Electrical Stimulation. Neuromodulation 7: 67-75

12. Lempka SF, Miocinovic S, Johnson MD, Vitek JL, McIntyre CC (2009) In vivo impedance spectroscopy of deep brain stimulation electrodes. J Neural Eng 6: 046001.

13. Satzer D, Lanctin D, Eberly LE, Abosch A (2014) Variation in deep brain stimulation electrode impedance over years following electrode implantation. Stereotact Funct Neurosurg 92: 94-102.

14. Simpson J, Ghovanloo M (2007) An Experimental Study of Voltage, Current and Charge Controlled Stimulation Front-End Circuitry in IEEE International Symposium on Circuits and Systems. ISCAS, New Orleans.

15. Butson CR, McIntyre CC (2005) Tissue and electrode capacitance reduce neural activation volumes during deep brain stimulation. Clin Neurophysiol 116: 2490-2500.

16. Grant PF, Lowery MM (2010) Effect of dispersive conductivity and permittivity in volume conductor models of deep brain stimulation. IEEE Trans Biomed Eng 57: 2386-2393.

17. Schmidt C, Grant P, Lowery M, van Rienen U (2013) Influence of uncertainties in the material properties of brain tissue on the probabilistic volume of tissue activated. IEEE Trans Biomed Eng 60: 1378-1387.

18. Aström M, Johansson JD, Hariz MI, Eriksson O, Wårdell K (2006) The effect 
Citation: Alonso F, Hemm-Ode S, Wårdell K (2015) Influence on Deep Brain Stimulation from Lead Design, Operating Mode and Tissue Impedance Changes - A Simulation Study. Brain Disord Ther 4:169. doi:10.4172/2168-975X.1000169

of cystic cavities on deep brain stimulation in the basal ganglia: a simulationbased study. J Neural Eng 3: 132-138.

19. Aström M, Lemaire JJ, Wårdell K (2012) Influence of heterogeneous and anisotropic tissue conductivity on electric field distribution in deep brain stimulation. Med Biol Eng Comput 50: 23-32.

20. Butson CR, Cooper SE, Henderson JM, McIntyre CC (2007) Patient-specific analysis of the volume of tissue activated during deep brain stimulation. Neuroimage 34: 661-670

21. Aström M, Zrinzo LU, Tisch S, Tripoliti E, Hariz MI, et al. (2009) Method for patient-specific finite element modeling and simulation of deep brain stimulation. Med Biol Eng Comput 47: 21-28.

22. Åström M, Tripoliti E, Hariz MI, Zrinzo LU, Martinez-Torres I, et al. (2010) Patientspecific model-based investigation of speech intelligibility and movement during deep brain stimulation. Stereotact Funct Neurosurg 88: 224-233.

23. Chaturvedi A, Butson CR, Lempka SF, Cooper SE, McIntyre CC (2010) Patientspecific models of deep brain stimulation: influence of field model complexity on neural activation predictions. Brain Stimul 3: 65-67.

24. Hemm S, Mennessier G, Vayssiere N, Cif L, Fertit HE, et al. (2005) Deep brain stimulation in movement disorders: stereotactic coregistration of twodimensional electrical field modeling and magnetic resonance imaging. $J$ Neurosurg 103: 949-955.

25. Vasques X, Cif L, Hess O, Gavarini S, Mennessier G, et al. (2009) Stereotactic model of the electrical distribution within the internal globus pallidus during deep brain stimulation. J Comput Neurosci 26: 109-118.

26. Wårdell K, Zrinzo L, Hariz M, Andersson M (2013) Patient-Specific Brain Modelling for Deep Brain Stimulation Simulation in 6th International IEEE EMBS Conference on Neural Engineering proceedings 148-151.

27. Wårdell $\mathrm{K}$, Kefalopoulou Z, Diczfalusy $\mathrm{E}$, Andersson $\mathrm{M}$, Åström $\mathrm{M}$, et al (2015) Deep brain stimulation of the pallidum internum for gilles de la tourette syndrome: a patient-specific model-based simulation study of the electric field. Neuromodulation 18: 90-96

28. Kuncel AM, Cooper SE, Grill WM (2008) A method to estimate the spatial extent of activation in thalamic deep brain stimulation. Clin Neurophysiol 119: 2148 2158.

29. Åström M, Diczfalusy E, Martens H, Wårdell K (2015) Relationship between Neural Activation and Electric Field Distribution during Deep Brain Stimulation. IEEE Trans Biomed Eng 62: 664-672.

30. Yousif N, Bayford R, Bain PG, Liu X (2007) The peri-electrode space is significant element of the electrode-brain interface in deep brain stimulation: a computational study. Brain Res Bull 74: 361-368.

31. Andreuccetti D, Fossi R, Petrucci C (2005) Dielectric properties of the tissue. Italian National Research Council, Institute for Applied Physics, Florence, Italy.
32. Yousif N, Bayford R, Liu X (2008) The influence of reactivity of the electrodebrain interface on the crossing electric current in therapeutic deep brain stimulation. Neuroscience 156: 597-606.

33. Schmidt C, van Rienen U (2012) Modeling the field distribution in deep brain stimulation: the influence of anisotropy of brain tissue. IEEE Trans Biomed Eng 59: 1583-1592.

34. Wårdell K, Diczfalusy E, Åström M (2011) Patient-Specific Modeling and Simulation of Deep Brain Stimulation. Studies in Mechanobiology, Tissue Engineering and Biomaterials 9: 357-375.

35. Nossek E, Korn A, Shahar T, Kanner AA, Yaffe H, et al. (2011) Intraoperative mapping and monitoring of the corticospinal tracts with neurophysiological assessment and 3-dimensional ultrasonography-based navigation. Clinical article. J Neurosurg 114: 738-746.

36. Limousin P, Tisch S (2009) DBS stimulator programming. In: Bain P (ed) Deep brain stimulation, Oxford university press. $65-71$.

37. Ohue S, Kohno S, Inoue A, Yamashita D, Harada H, et al. (2012) Accuracy of diffusion tensor magnetic resonance imaging-based tractography for surgery of gliomas near the pyramidal tract: a significant correlation between subcortical electrical stimulation and postoperative tractography. Neurosurgery 70: 283293.

38. Seidel K, Beck J, Stieglitz L, Schucht P, Raabe A (2013) The warning-sign hierarchy between quantitative subcortical motor mapping and continuous motor evoked potential monitoring during resection of supratentorial brain tumors. J Neurosurg 118: 287-296.

39. Haberler C, Alesch F, Mazal PR, Pilz P, Jellinger K, et al. (2000) No tissue damage by chronic deep brain stimulation in Parkinson's disease. Ann Neurol 48: $372-376$.

40. Nielsen MS, Bjarkam CR, Sørensen JC, Bojsen-Møller M, Sunde NA, et al. (2007) Chronic subthalamic high-frequency deep brain stimulation in Parkinson's disease--a histopathological study. Eur J Neurol 14: 132-138.

41. Schmidt C, van Rienen U (2012) Quantification of uncertainties in brain tissue conductivity in a heterogeneous model of deep brain stimulation using a nonintrusive projection approach. Conf Proc IEEE Eng Med Biol Soc 2012: 4136 4139 .

42. Howell B, Naik S, Grill WM (2014) Influences of interpolation error, electrode geometry, and the electrode-tissue interface on models of electric fields produced by deep brain stimulation. IEEE Trans Biomed Eng 61: 297-307.

43. McIntyre CC, Mori S, Sherman DL, Thakor NV, Vitek JL (2004) Electric field and stimulating influence generated by deep brain stimulation of the subthalamic nucleus. Clin Neurophysiol 115: 589-595. 